\title{
La imposición indirecta de transacciones digitales. La porción que nos falta recaudar en tiempos de crisis
}

\section{The indirect imposition of digital transactions. The portion that we need to collect in times of crisis}

\section{Tributação indireta das transaçoës digitais. A parte que precisamos coletar em tempos de crise}

ISAÁc G. Arias E.* RAÚL ZAMBRANO**

Contador público; magíster en Dirección de Finanzas, programa conjunto de la Universidad del Salvador, Argentina, y State University of New York. Director de Cooperación y Tributación Internacional del Centro Interamericano de Administraciones Tributarias. garias@ciat.org / https://orcid.org/00000002-9092-9272.

** Ingeniero de sistemas. Director de Asistencia Técnica y TIC del Centro Interamericano de Administraciones Tributarias.rzambrano@ciat.org / https:// orcid.org/0000-0002-2658-7992.

DOI: https://doi.org/10.18601/16926722.n18.01 


\section{Resumen}

El presente artículo analiza los riesgos de la economía digital en la tributación indirecta, con especial énfasis en los mecanismos de recaudo, en atención al papel fundamental que desempeñan en época de crisis como la que estamos viviendo actualmente a nivel mundial por el covid-19.

Palabras clave: covid-19; economía digital; imposición directa; mecanismo de recaudo y acción 1 BEPS.

\section{Abstract}

This article analyzes the risks of the digital economy in indirect taxation, with special emphasis on collection mechanisms, in response to the fundamental role they play in times of crisis such as the one we are currently experiencing worldwide due to the new coronavirus Covid- 19.

Key words: Covid-19; digital economy; direct taxation; collection mechanism and action 1 BEPS.

\section{Resumo}

Este artigo analisa os riscos da economia digital na tributação indireta, com ênfase especial nos mecanismos de cobrança em resposta ao papel fundamental que desempenham em tempos de crise, como o que estamos enfrentando atualmente em todo o mundo devido ao novo coronavírus Covid- 19.

Palavras-chave: Covid-19; economia digital; tributação direta; mecanismo de cobrança e ação 1 BEPS. 


\section{Introducción}

El Centro Interamericano de Administraciones Tributarias (CIAT), desde el inicio de la pandemia por el covid-19, monitorea la situación económica, social y sanitaria de la región, con el propósito de definir posibles recomendaciones para acciones inmediatas y futuras relacionadas con la gestión de los ingresos públicos.

A pesar de que la crisis no ha concluido, los números muestran un escenario crítico. De acuerdo con el informe de movilidad de Google, los desplazamientos a lugares de trabajo se redujeron en un $47,5 \%$, los desplazamientos a tiendas y lugares de ocio se afectaron en un 54,3\%, los desplazamientos a estaciones de tránsito se redujeron en un $57 \%$. Cabe destacar que estos datos se refieren a la media. En algunos de los países más afectados por la pandemia y que impusieron las medidas de confinamiento y distanciamiento social más estrictas, el impacto ha sido mayor (Díaz de Sarralde, 2020). Ello se refleja en el PIB $\mathrm{y}$, consecuentemente, en los ingresos públicos, en un momento en el cual, en la mayoría de los casos, el rol de los Estados toma relevancia en la difícil tarea de mantener y reconstituir la economía.

En lo que a ingresos públicos respecta, algunas de las preguntas que actualmente se formulan todos los países son: ¿qué sectores económicos o negocios no se han visto afectados o se han beneficiado de esta situación? ¿Qué sectores económicos o negocios requieren apoyo para subsistir? ¿Para qué sectores económicos o negocios la crisis actual representa un problema coyuntural? ¿Para qué sectores económicos o negocios la crisis les deja un problema estructural? Finalmente, la gran pregunta es: ¿qué tratamiento sería adecuado para cada uno de estos casos?

Análisis económicos y financieros asociados a un eficiente y efectivo sistema de gestión de riesgos de incumplimiento tributario pudieran ser instrumentos clave para monitorear la situación de los negocios e individuos, e identificar acciones de tratamiento. Sin embargo, no es el objetivo de este artículo responder en su totalidad las referidas preguntas. Su propósito es tratar aspectos específicos relacionados con la tributación de la economía digital.

Este sector de la economía, que por distintas razones se ha encontrado al margen de los sistemas de tributación, constituye una oportunidad para asegurar ingresos tributarios.

La economía digital presenta grandes riesgos. Entre ellos se destacan, la no declaración de ingresos, los trabajadores que no cotizan a la seguridad social, el desequilibrio económico que puede afectar de manera significativa la economía tradicional y dejar trabajadores socialmente desprotegidos. Estos riesgos son muy difíciles de identificar con los actuales sistemas de muchas administraciones tributarias. Ejemplos específicos de situaciones que se derivan de la economía digital serían: el uso de páginas web en más de un dominio para desviar pagos a diversas cuentas, uso de marketplaces en el exterior para simular transacciones internacionales, fraccionamiento de ventas entre varias terminales virtuales, no declaración de publicidad generada bajo sistemas de afiliación, no declaración de ingresos por parte de influencers que promocionan bienes y servicios, no 
declaración de transacciones que se llevan a cabo por medio de subastas donde no existe contacto entre las partes, entre otros.

A esto se suma la posibilidad del uso de monedas cifradas como medios alternos de pago, eliminando o limitando significativamente la participación de intermediarios financieros, y, con ello, la capacidad de contar con puntos de control o de obtención de información.

Los nuevos modelos de negocio abren espacios para los consumidores, pero con esas oportunidades vienen también amenzas, entre ellas, la posibilidad de la quiebra de compañías y la pérdida de empleos, el traslado de las operaciones comerciales fuera de la jurisidicción y la consecuente pérdida de los tributos derivados directa o indirectamente de esas actividades.

Para abordar de manera general los riesgos que presenta la economía digital hace falta un cambio de cultura organizacional, modificaciones significativas a los sistemas tributarios, inversión en infraestructura y recursos humanos. Ello no se dará de inmediato. Sin embargo, las necesidades actuales de los países requieren tomar medidas oportunas para mantener la recaudación, mientras en paralelo se preparan para los desafíos del largo plazo. Por esta razón, nos enfocaremos en un nuevo desarrollo que a nuestro entender constituye una oportunidad para asegurar en el mediano plazo una porción de ingresos tributarios que la mayoría de los países en desarrollo -y algunos desarrollados- no están percibiendo, de un sector de la economía que, en términos generales, no ha sido afectado por la crisis y que en ciertos casos se ha visto beneficiado.

En particular, nos referimos a los mecanismos para gestionar impuestos indirectos -principalmente el IVA- generados por negocios que operan en la economía digital y que no tienen presencia física en el país donde ofrecen sus productos y servicios.

\section{Mecanismo de recaudo de impuestos indirectos generados por negocios internacionales}

La Organización para la Cooperación y el Desarrollo Económicos (OCDE) (2017), en el seno del Working Party No. 9, que discute aspectos relacionados con los impuestos al consumo, y en el marco de la Acción 1 del Plan de Acción BEPS, que trata los desafíos que en el ámbito de la tributación trae la economía digital, desarrolló una propuesta sobre cómo gravar los impuestos indirectos cuando los bienes y servicios son brindados por negocios digitales que no tienen presencia física en el destinatario de estos. Ejemplo de negocios digitales son el comercio electrónico, los denominados "negocios de aplicaciones" - application stores-, la publicidad en línea, la computación en la nube, las plataformas de red colaborativas, la prestación de servicios a través de internet, la comercialización de intangibles o los servicios de pago en línea, entre otros.

El mecanismo propuesto en los documentos de la $\operatorname{OEDC}(2017 ; 2019)$ consiste en utilizar una plataforma digital que permita a las empresas de la economía digital registrarse en los países donde comercializa sus bienes y servicios mediante un procedimiento 
simplificado, para luego declarar sus operaciones e ingresar el impuesto correspondiente, todo ello sin necesidad de mantener presencia física en los países destinatarios de sus bienes y servicios.

En términos generales, los principales aspectos por considerar para implementar plataformas digitales para gestionar impuestos indirectos en este escenario son:

Evaluar las normas de los impuestos indirectos por considerar y el código tributario - $\mathrm{u}$ otras normas que correspondan-, a efectos de ajustar normas legales o normas secundarias o reglamentarias, así como procedimientos administrativos relacionados con el registro, la declaración, el pago y el control -p. ej.: regímenes de información y procesos internos de verificación-.

Considerando que este tipo de sistemas opera para contribuyentes que no tienen presencia física en el país donde llevan a cabo sus operaciones, es muy importante definir cuál va a ser el "hecho imponible" que lleve a gravar la operación. Por ejemplo: jurisdicción donde se consume o aprovecha el bien o servicio, jurisdicción desde donde se efectúa el pago, jurisdicción donde se encuentra domiciliado el comprador, criterios combinados, otros. La definición de estos criterios condiciona la forma de declarar transacciones y las estrategias de control.

Las plataformas digitales consideran todas las obligaciones relacionadas con impuestos indirectos - principalmente IVA, lo cual dependerá del sistema tributario y voluntad de cada país-, en particular el registro y la declaración. El pago podría llevarse a cabo por medio de otros sistemas disponibles, siempre que permitan efectuar pagos desde el exterior. La posibilidad de considerar impuestos subnacionales es un asunto que cabe analizar para algunos países, pero que agregaría complejidad al sistema.

Deberían considerarse mecanismos para evitar doble imposición. Sin embargo, es necesario tener en cuenta que dichos mecanismos pueden ocasionar fraudes, debiendo existir controles basados en la cooperación internacional entre administraciones tributarias ( $\mathrm{p}$. ej., intercambio internacional de información cuando un contribuyente solicita un crédito o exención).

Es necesario considerar la emisión de comprobantes que permitan a los consumidores locales respaldar sus operaciones para considerar el impuesto en sus diferentes fases dentro de la cadena de valor, y en su caso deducirlas de otros impuestos. Dichos comprobantes deberían tener un diseño simplificado, pero que permita un control efectivo y oportuno. Asimismo, sería ideal que la administración tributaria pueda recibirlos cuando se genera la operación o, de no ser posible, periódicamente. Puede que la tecnología blockchain, en un modelo de consorcio privado, permita generar un proceso donde intervengan todas las partes de la transacción, que asegure a las administraciones tributarias que el $100 \%$ de las operaciones documentadas equivale al $100 \%$ de las operaciones efectuadas.

En este sentido, para cada tipo de negocio digital alcanzado por este mecanismo especial de registro, declaración y pago, resulta necesario definir procedimientos de control para evaluar la veracidad de la información presentada en la declaración, considerando información de diversas fuentes, por ejemplo, facturación o comprobante de la transacción, 
medios de pago, direcciones IP, información internacional, deducciones de clientes en sus declaraciones de renta, entre otros.

Es importante que el procedimiento completo de registro y declaración sea simplificado, para motivar el cumplimiento por parte de los negocios digitales y que estos procedimientos puedan ser armonizados para generar certeza a las empresas sobre como cumplir con obligaciones tributarias a un costo razonable en diversos países. Ello nos lleva a que la implementación de este mecanismo, para que sea efectivo, debe llevarse a cabo de manera coordinada, al menos a nivel regional; es ideal que la plataforma pueda permitir un solo procedimiento de registro para cumplir con obligaciones tributarias en varios países.

\section{Panorama en algunos países de América Latina}

Con el ánimo de obtener ingresos tributarios de negocios digitales, a nivel global muchos países optaron por tomar medidas unilaterales, cuyos resultados han sido positivos en términos absolutos, pero tal vez mejorables en términos relativos; para ello, han sido necesarias en la mayoría de los casos acciones que aborden el tema de manera más estructural. Por lo general, las medidas adoptadas han hecho uso de medios de pago para lograr su propósito recaudatorio. A continuación, se detallan brevemente algunas experiencias de países de América Latina.

En Argentina, los servicios prestados por negocios digitales no residentes y consumidos con sede en Argentina son gravados por el impuesto al valor Agregado (IVA), y es aplicable la tasa general del impuesto, que es del 21\%. La Administración Federal de Ingresos Públicos de Argentina (AFIP) ha compilado una lista de servicios digitales que se ven afectados por las nuevas reglas (p. ej.: servicios de streaming content). Para definir si el servicio fue consumido en Argentina, la AFIP verifica la tarjeta SIM del teléfono celular que recibe el servicio, la dirección IP, la dirección de facturación del destinatario y la cuenta bancaria utilizada para el pago o la dirección de la tarjeta de crédito.

En México se brinda a los residentes nacionales y extranjeros que permitan el uso de plataformas tecnológicas a personas físicas que presten de forma independiente el servicio de transporte terrestre de pasajeros o entrega de alimentos preparados, la opción de retener el impuesto sobre la renta y el IVA generado por las transacciones de dichas personas físicas, efectuadas por medio de tales plataformas.

En Chile se aplica el IVA a los servicios digitales B2B (negocio a negocio) y B2C (negocio a consumidor), siguiendo las recomendaciones realizadas por la oCDE, de manera similar al procedimiento descrito en el apartado anterior.

En Colombia existe un mecanismo de recaudo combinado. Para las operaciones B2B se estableció un mecanismo de retención en la fuente sobre los sujetos que pertenecen al régimen común del IVA. Para las operaciones $\mathrm{B} 2 \mathrm{C}$ se estableció un régimen de recaudo basado en el registro, la declaración y el pago por parte de los prestadores de servicios que operan desde el exterior. Sin embargo, en las operaciones B2C también se estableció un mecanismo de retención en la fuente a cargo de las entidades emisoras de tarjetas débito 
y crédito, vendedores de tarjetas prepago, recaudadores de efectivo a cargo de terceros y los demás que designe la administración de impuestos. La retención aplica a los siguientes servicios electrónicos o digitales, cuando el proveedor del servicio se acoja voluntariamente a este sistema alternativo de pago del impuesto:

a. Suministro de servicios audiovisuales.

b. Servicios prestados a través de plataformas digitales.

c. Publicidad online.

d. Enseñanza y entrenamiento a distancia.

e. Suministro de derechos de uso o explotación de intangibles.

f. Otros servicios electrónicos o digitales con destino a usuarios ubicados en Colombia.

Los prestadores de servicios electrónicos digitales podrán acogerse voluntariamente al sistema de retención. La administración tributaria indica de manera taxativa el listado de prestadores desde el exterior a los que deberá practicárseles la retención, siempre que estos:

a. Presten sus servicios a usuarios en Colombia.

b. No se hayan acogido al sistema de declaración bimestral de IVA y se acojan voluntariamente a este sistema.

c. El valor cobrado a los usuarios corresponda a la base gravable del IVA por los servicios electrónicos.

En Costa Rica, la norma establece la posibilidad de que la Administración Tributaria realice el cobro del impuesto por medio de proveedores o intermediarios extranjeros que ponen a disposición de un consumidor final bienes intangibles o servicios digitales. La Administración Tributaria dispone las condiciones por las cuales los proveedores e intermediarios deben cumplir con estas obligaciones. Adicionalmente, se establece la percepción del Iva por medio de las entidades que emitan tarjetas de crédito o débito cuando sus tarjetahabientes utilicen este medio de pago para adquirir servicios por medio de internet o cualquier otra plataforma digital y consumirlos en el territorio nacional.

Como se desprende de los párrafos anteriores, Chile presenta una de las experiencias más avanzadas, al adoptar las propuestas de la OCDE, mientras que otros países han considerado aproximaciones a estas recomendaciones o medidas unilaterales haciendo uso de medios de pago, lo cual podríamos considerar como medidas de transición, entendiendo que a futuro esperamos que se adopte el uso de plataformas digitales. En recientes actividades internacionales, los principales actores de la economía digital han manifestado su interés en que exista una plataforma armonizada y con procedimientos simplificados que les permita cumplir con sus obligaciones tributarias en tiempo y forma. 


\section{Iniciativas internacionales con enfoque en países en desarrollo o de bajos ingresos}

La oCDE, con el apoyo del Banco Mundial, el Banco Interamericano de Desarrollo (BID) y el Centro Interamericano de Administraciones Tributarias (CIAT) se encuentra trabajando en la elaboración de un manual o toolkit orientado a países en desarrollo -especialmente de América Latina y el Caribe-, sobre cómo implementar procedimientos para que las empresas digitales cumplan sus obligaciones tributarias relacionadas con impuestos indirectos, haciendo uso de una plataforma digital.

Se entiende que existe un consenso sobre el criterio de destino en la aplicación de derechos impositivos de impuestos indirectos, particularmente el IVA. Sin embargo, no todos los países contemplan las condiciones para gravar las operaciones resultantes del desarrollo de modelos de negocio altamente informatizados sin ninguna presencia física. Es posible que la responsabilidad del pago de esas obligaciones recaiga en el consumidor final, que debería consignar el IVA relacionado con una operación directamente al tesoro nacional o que la operación no esté gravada en absoluto. No es fácil imaginar que cada uno de los usuarios de un servicio de transmisión de música o videos en un país realice una declaración mensual del IVA que debería pagar por ese servicio, junto con el pago. La opción de que la empresa se registre localmente en un país, cumpliendo los requisitos estándares establecidos para un contribuyente local, se enfrenta directamente con la premisa de este tipo de operaciones.

Contar con un conjunto de guías para ajustar la legislación y los procedimientos bajo un modelo común, a diferencia de medidas unilaterales que por su propia característica pueden ser muy diversas, mejora el ambiente para los diferentes actores. Por un lado, los Estados saben que el modelo adoptado facilita el acceso a esos derechos tributarios de una manera que no puede ser considerada arbitraria por las empresas que operan en ese entorno. Por el otro, las empresas actúan bajo un modelo estándar compartido por muchos países y evitan la necesidad de especializar su capacidad de cumplimiento tributario de país en país.

Por su parte, el CIAT, en el Marco del Programa de Cooperación CIAT-NORAD (Norwegian Agency for Development Cooperation) se encuentra desarrollando una plataforma digital de fuente abierta que operará en la nube y que será un bien público internacional. Esta plataforma digital permitirá implementar muchas de las recomendaciones existentes en los documentos de la OCDE y, en particular, del referido anual, ya que la plataforma digital está orientada a países en desarrollo o de bajos ingresos. Actualmente, se encuentra trabajando un equipo conformado por expertos en impuestos indirectos, tecnología, tributación internacional y funcionarios de administraciones tributarias de América Latina que acompañan la iniciativa. Asimismo, se estableció un canal de comunicación y retroalimentación con el equipo de trabajo conformado para elaborar el toolkit antes mencionado. Se estima que la misma estará disponible en 2021. 
El desarrollo de esta solución, que cada país que decida adoptar por su propia cuenta, permitirá a cada administración tributaria facilitar el acceso a los recursos rápidamente, sin necesidad de desarrollar módulos nuevos en su sistema de información, pero, sobre todo, que la interfaz de ese sistema es ya conocida y utilizada por las empresas multinacionales. Esto disminuirá los requerimientos de interacción de las administraciones con las empresas para divulgar formatos, catálogos o procedimientos. Las administraciones podrán determinar si van a instalar el sistema en centros de datos propios, o si van a producir el sistema desde una plataforma de nube. Esta versatilidad permitirá a que jurisdicciones de diferente tamaño, con administraciones con distintas capacidades tecnológicas puedan utilizar el sistema.

Las empresas, en cambio, evitarán interactuar con una infinidad de aplicaciones y métodos en diferentes mercados, utilizando una herramienta e interfaz común. La parametrización del sistema debe facilitar adaptarse sin grandes costes a las particularidades de cada jurisdicción.

\section{Ventajas de los mecanismos para gestionar impuestos indirectos originados por negocios que operan en la economía digital}

Las plataformas digitales permiten generar "nuevos" ingresos tributarios por medio de eficiencia administrativa, sin necesidad de recurrir al incremento de la carga tributaria por medio de reformas a la estructura de los tributos existentes (base imponible o tasa) o a la creación de nuevos, lo cual es complejo y en algunos casos no deseable por el impacto que puedan generar en el actual contexto -efectos en la inversión, definición adecuada de sujetos para no perjudicar a los afectados, etc.-. El margen para incrementar la carga tributaria por medio de la eficiencia administrativa es elevado en países en desarrollo, donde las brechas tributarias en los principales impuestos suelen tener dos dígitos.

Los impuestos indirectos no solo son los que representan una mayor porción de la recaudación de la mayoría de los países en desarrollo - por ejemplo, en el año 2017 el 27,6 \% del total de ingresos de América Latina y el Caribe se originó en el IVA, mientras que el $22,8 \%$ en otros impuestos indirectos sobre bienes y servicios-, sino que están ligados directamente al nivel de actividad independientemente de la situación que presenten los negocios (OCDE, UN-ECLA, CIAT y BID, 2020). Ello permite a los Estados percibir ingresos ante la gradual recuperación de la economía, lo que no sucedería en el caso del impuesto a la renta, donde las pérdidas suelen compensarse con utilidades futuras, lo que transforma, por ejemplo, los impuestos a las ventas o el IVA en oportunidades de ingreso en tiempos de recuperación económica -e incluso durante la crisis-. Con mayor razón en los negocios digitales donde se crea una nueva fuente de ingreso tributario en el marco de impuestos existentes.

El impuesto a la renta también constituye una oportunidad de generar ingresos tributarios no obtenidos hasta el momento en este ámbito de actuación-economía digital-; sin embargo, de acuerdo con las discusiones que se mantienen a nivel internacional en el seno 
de la OCDE y con el respaldo del G20, es necesario un plan de acción consistente y armonizado que requiere profundas adecuaciones de la política tributaria y considerables esfuerzos administrativos. A la fecha no existe un consenso sobre las propuestas formuladas, lo que lleva a los países a plantearse la disyuntiva de implementar medidas unilaterales para asegurar la recaudación inmediata -lo que puede generar efectos nocivos-o esperar a que se cree un consenso internacional para luego avanzar sobre un extenso y complejo mecanismo de implementación. Por ello es que los impuestos indirectos representan una alternativa más oportuna para los Estados.

Por medio de los procedimientos para recaudar impuestos indirectos por transacciones digitales se lograría generar mayor certeza entre empresas de la economía digital sobre cómo cumplir con obligaciones tributarias, asegurar la recaudación de recursos que actualmente no se obtienen, permitir a los clientes de negocios digitales -en la relación B2B- obtener documentos válidos para deducir costos y gastos por bienes y servicios adquiridos, lo que se traduciría en un mejor control y una mayor equidad entre la situación de los negocios que operan en la economía tradicional y los negocios digitales, al equiparar por medio del cobro de impuestos sus precios o utilidades.

\section{Conclusiones}

En el escenario actual, donde los gobiernos del mundo ven cómo se desmoronan las economías y, por ende, las bases imponibles de los principales impuestos, el desarrollo al que nos referimos constituye, a nuestro entender, una de las medidas más trascendentes por implementar en el corto o mediano plazo. Las ventajas están a la vista: permite obtener recursos tributarios de negocios que se han visto favorecidos, genera equidad al proteger a la economía tradicional de las distorsiones originadas por diferencias en la carga tributaria, facilita a las empresas de la economía digital alcanzar niveles de cumplimiento en las distintas jurisdicciones sin incurrir en costos de cumplimiento desproporcionados, lo que mejora su reputación y potencia sus relaciones con clientes y operadores, en especial las relaciones $\mathrm{B} 2 \mathrm{~B}$, donde los costos y gastos deben ser formalizados a fin de ser deducidos para efectos contables y tributarios.

\section{Referencias}

Díaz de Sarralde, S. (2020). ¿Cómo pagar las consecuencias de una pandemia? Una primera aproximación a las analogías del pasado y las alternativas para el futuro. Centro Interamericano de Administraciones Tributarias (СIAT). https://www.ciat. org/Biblioteca/DocumentosdeTrabajo/2020/DT_01_2020_sarralde.pdf

Organisation for Economic Co-operation and Development (OECD) (2017). Mechanisms for effective collection of VAT/GST When the Supplier is not Located in the Jurisdiction of Taxation. OECD. 
Organisation for Economic Co-operation and Development (OECD) (2019). The Role of Digital Platforms in the Collection of VAT/GST on On-Line Sales. OECD.

Organización para la Cooperación y el Desarrollo Económicos (OCDE), Comisión Económica para América Latina y el Caribe (UN-ECLA), Centro Interamericano de Administraciones Tributarias (CIAT) y Banco Interamericano de Desarrollo (BID). (2020). Revenue Statistics in Latin America and the Caribbean.

Fecha de recepción: 15 de junio de 2020

Fecha de aprobación par 1: 02 de septiembre de 2020

Fecha de aprobación par 2: 15 de julio de 2020 